\title{
Hydrogen-Assisted Laser-Induced Resonant Transitions between Metastable States of Antiprotonic Helium Atoms
}

\author{
B. Ketzer, ${ }^{1}$ F. J. Hartmann, ${ }^{1}$ T. von Egidy, ${ }^{1}$ C. Maierl, ${ }^{1}$ R. Pohl,${ }^{1}$ J. Eades,${ }^{2}$ E. Widmann, ${ }^{2}$ T. Yamazaki, ${ }^{2}$ \\ M. Kumakura, ${ }^{3}$ N. Morita, ${ }^{3}$ R. S. Hayano, ${ }^{4}$ M. Hori,${ }^{4}$ T. Ishikawa, ${ }^{4}$ H. A. Torii, ${ }^{4}$ I. Sugai, ${ }^{5}$ and D. Horváth ${ }^{6}$ \\ ${ }^{1}$ Physik-Department, Technische Universität München, D-85747 Garching, Germany \\ ${ }^{2}$ CERN, CH-1211 Geneva 23, Switzerland \\ ${ }^{3}$ Institute for Molecular Science, Myodaiji, Okazaki 444, Japan \\ ${ }^{4}$ Department of Physics, University of Tokyo, 7-3-1 Hongo, Bunkyo-ku, Tokyo 113, Japan \\ ${ }^{5}$ Institute for Nuclear Study, University of Tokyo, 3-2-1 Midori-cho, Tanashi, Tokyo 188, Japan \\ ${ }^{6}$ Central Research Institute for Physics, Research Institute for Particle and Nuclear Physics, H-1525 Budapest, Hungary
}

(Received 21 October 1996)

\begin{abstract}
Laser resonance transitions between normally metastable states of antiprotonic helium atoms were observed making use of state dependent quenching effects caused by small admixtures of $\mathrm{H}_{2}$ molecules. By selectively shortening the lifetimes of states with higher principal quantum number $n$ as compared to those of lower $n$, this method for the first time provides access to all initially populated metastable states of $\bar{p} \mathrm{He}^{+}$atoms. This was demonstrated by observing the transitions $(n, l)=(38, l) \rightarrow(39, l+1)$, $l=35,36,37$ and $(n, l)=(37, l) \rightarrow(38, l+1), l=34,35,36 . \quad$ [S0031-9007(97)02579-9]
\end{abstract}

PACS numbers: 36.10.-k, 34.90.+q, 42.62.Fi

In a series of laser spectroscopy experiments on antiprotonic helium atoms $[1-6]$ we have shown that the unusual longevity of some $3 \%$ of antiprotons stopped in helium [7$12]$ is due to the formation of a metastable class of these $\bar{p} \mathrm{He}^{+}\left(\equiv \bar{p}-e^{-}-\mathrm{He}^{2+}\right)$ atoms. Our experiments indicate that members of this class occupy states with principal quantum number $n \sim n_{0} \equiv \sqrt{M^{*} / m_{e}} \simeq 38\left(M^{*}\right.$ denoting the reduced mass of the $\bar{p}^{4} \mathrm{He}^{2+}$ system and $m_{e}$ the electron mass) and angular momentum quantum number $l \leqq n-1$, thereby confirming the long-held assumption that the $\bar{p}$ initially occupies a state with spatial distribution and binding energy similar to that of the electron it displaces.

The experimental technique used until now, however, was restricted to laser-stimulated transitions between metastable states $(n, l)$ and adjacent nonmetastable ones $(n-1, l-1)$ at the end of each cascade with vibrational quantum number $v=n-l-1=$ const [13]. We have now overcome this restriction by taking advantage of state-dependent quenching effects observed when $\mathrm{H}_{2}$ is added to the helium at ppm levels. By selectively shortening the lifetimes of states with higher principal quantum number $n$, a further region of the $(n, l)$ plane can be accessed by our laser resonance technique. The present paper reports observation of six laser-induced transitions by this method.

Even before we began laser spectroscopy experiments we had observed a particularly sensitive dependence of the delayed annihilation time spectrum (DATS) on the presence of $\mathrm{H}_{2}$ molecules [8,9,12]. At concentrations as low as a few ppm, these clearly converted certain long-lived states to shorter-lived ones, resulting in the appearance of a fast-decaying component in the DATS together with a corresponding reduction of the long-lived remainder.
In the laser experiments the sharp increase in annihilation rate that accompanies the stimulated transitions from metastable to Auger-dominated nonmetastable levels serves as the indicator of the resonance condition [13]. This technique offered the means to investigate the effect of $\mathrm{H}_{2}$ on the lifetime of individual metastable $\bar{p} \mathrm{He}^{+}$levels rather than on the overall DATS, as is reported in [14].

We began these studies by examining the already well known transitions $(n, l)=(37,34) \rightarrow(36,33)$ in the cascade $v=2(470.724 \mathrm{~nm})$ and $(n, l)=(39,35) \rightarrow$ $(38,34)$ in the cascade $v=3(597.259 \mathrm{~nm})$, both between a long-lived and a short-lived level. The experimental arrangement was similar to the one used in previous laser spectroscopy experiments [1-3]. For the present experiments with $\mathrm{H}_{2}$ admixtures we used ${ }^{4} \mathrm{He} 5.6$ (99.9996\% pure, i.e., impurities $\leq 4 \mathrm{ppm}$ ) to which small amounts of $\mathrm{H}_{2} 5.0$ ( $99.999 \%$ pure, i.e., impurities $\leq 10 \mathrm{ppm}$ ) had been premixed at ppm levels with a relative accuracy of $2 \%$. To assure that the target gas was not contaminated by impurities such as $\mathrm{O}_{2}$ or $\mathrm{N}_{2}$ it was kept at a temperature of $30 \mathrm{~K}$ throughout the experiment. A detailed description of the experimental and evaluation procedures will be given in [15].

First, the resonance intensity of the $470.724 \mathrm{~nm}$ transition $(n, l)=(37,34) \rightarrow(36,33)$ was measured as a function of the laser timing for various $\mathrm{H}_{2}$ admixtures. When plotted against the laser firing time, the resonance intensity of a single laser pulse shows an approximately single exponential decrease with a lifetime $T$. This "overall cascade lifetime" tagged by the laser resonance not only reflects the population and lifetime of the resonantly deexcited state, but also those of higher lying states in the same cascade feeding this state. Second, the lifetime $\tau$ of the resonantly deexcited state $(37,34)$ alone was determined by measuring the time constant with which the 
DATS returns after laser irradiation to its original form. This "depletion-recovery" method constitutes a modelindependent way to determine the lifetime of the deexcited metastable state directly [16].

Figure 1(a) shows $\tau(37,34)$ together with $T_{v=2}$ as a function of the $\mathrm{H}_{2}$ admixture ratio. Already at $30 \mathrm{ppm}$ $\mathrm{H}_{2}$ admixture the overall cascade lifetime $T_{v=2}$ decreased to $1.38 \pm 0.04 \mu \mathrm{s}$, about $700 \mathrm{~ns}$ shorter than the value in pure helium. On the other hand, the lifetime $\tau(37,34)$ of the last metastable state in the cascade remained nearly unchanged $(0.99 \pm 0.04 \mu \mathrm{s})$, indicating that feeding from states above $(37,34)$ gradually decreases with increasing $\mathrm{H}_{2}$ admixture. At $300 \mathrm{ppm}_{2} T_{v=2}$ approached $\tau(37,34)$, showing that at $\mathrm{H}_{2}$ admixtures higher than $300 \mathrm{ppm}$ the resonance intensity of the $470.724 \mathrm{~nm}$ transition results only from the population of the last metastable state.

A different picture arose from the observation of the transition $(n, l)=(39,35) \rightarrow(38,34)$ for the cascade with $v=3$, summarized in Fig. 1(b). The overall cascade lifetime $T_{v=3}$ approached the individual lifetime of the last metastable state already at $10 \mathrm{ppm}_{2}$ admixture, indicating negligible contributions from states with $n>39$. At an $\mathrm{H}_{2}$ concentration of $100 \mathrm{ppm}$ the resonance spike was no longer visible.

We conclude from these observations that for the $v=$ 2 and $v=3$ cascades states with higher $n$ are much more strongly quenched by collisions with $\mathrm{H}_{2}$ molecules than those with lower $n$. For adjacent levels in these cascades, this effect leaves a higher population in the

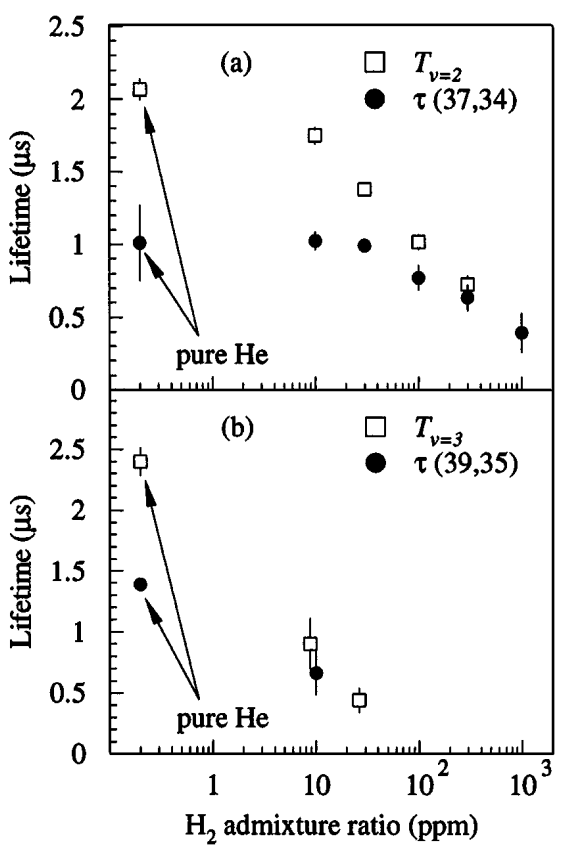

FIG. 1. Overall cascade lifetime $T$ for the cascades (a) $v=2$ and (b) $v=3$ as a function of the $\mathrm{H}_{2}$ concentration, compared with the single level lifetime $\tau$ of the last metastable state in the respective cascade, revealing a much stronger quenching of states with higher principal quantum number $n$. lower level than in the higher one, although the lower level lifetime has itself been reduced to some extent. A similar tendency is expected for other cascades $(v=1$ and 0 ). The laser resonance method can then be used to stimulate an "inverse" transition $(n, l) \rightarrow(n+1, l+1)$ between such normally metastable but $\mathrm{H}_{2}$-induced shortlived levels, provided the light pulse can be delivered early enough after the $\bar{p}$ stop.

This condition could be met by extracting the antiprotons from LEAR in short (100 ns) bunches, the signal that initiated bunched extraction being used to trigger the laser system in advance of the arrival of the bunch. In this way the $\bar{p} \mathrm{He}^{+}$atoms could be probed at times down to $180 \mathrm{~ns}$ after the instant of their formation.

Evidently it is not possible in the bunched mode to resolve individual annihilations of some $10^{5}-10^{6}$ antiprotons occurring over $10 \mu \mathrm{s}$. We therefore used the following "analog" method to measure the DATS: The Čerenkov light produced by the charged annihilation products in a simple Lucite radiator was detected by a fast gatable photomultiplier, and the resulting anode current was recorded by a digital oscilloscope. This technique was already used in $[6,17]$ and analog DATS starting at $500 \mathrm{~ns}$ were measured. In the present experiment we used a new gated PMT (R5504GX) which was developed by Hamamatsu Photonics Ltd. for this particular purpose. In this way we obtained distortionless analog DATS immediately after the end of the $\bar{p}$ pulse.

The first candidate for an " $\mathrm{H}_{2}$-assisted inverse resonance," abbreviated as "HAIR," was the transition $(n, l)=(38,35) \rightarrow(39,36) \quad(v=2)$ next to the well known $597.259 \mathrm{~nm}$ transition $(39,35) \rightarrow(38,34)$ from a metastable to a short-lived state, since we expected that the $(39,36)$ state might be quenched by $\mathrm{H}_{2}$ admixtures as rapidly as the $(39,35)$ state. Figure 2 shows a series of analog DATS obtained at different laser wavelengths near the expected transition wavelength at $30 \mathrm{~K}, 5$ bars, and an $\mathrm{H}_{2}$ concentration of $100 \mathrm{ppm}$. As the laser wavelength approached $597.297 \mathrm{~nm}$ a small but significant peak appeared in the time spectrum, indicating an increased annihilation rate at exactly the laser firing time $(\sim 180 \mathrm{~ns}$ after the arrival of the $\bar{p}$ pulse). This slowly decaying spike in the DATS reflects the lifetime of the $(39,36)$ state, which was shortened to $\sim 40 \mathrm{~ns}$ by the $100 \mathrm{ppm} \mathrm{H}_{2}$ admixture, as expected. For the normal metastable to short-lived state transition $(39,35) \rightarrow(38,34)$ the spike was much sharper, as shown in the lowest panel of Fig. 2.

The resonance profile for the transition $(n, l)=$ $(38,35) \rightarrow(39,36)$, shown in Fig. 3(a), was obtained by plotting the background subtracted area under the peak, normalized to the area under the spectrum between $150 \mathrm{~ns}$ and $2 \mu \mathrm{s}$, versus the vacuum wavelength of the laser light. Fitting a Lorentzian function convoluted with a Gaussian, which takes into account the laser bandwidth of $7 \mathrm{pm}$, to the data, the central vacuum wavelength for this transition was found to be $597.298 \pm 0.002 \mathrm{~nm}$. 


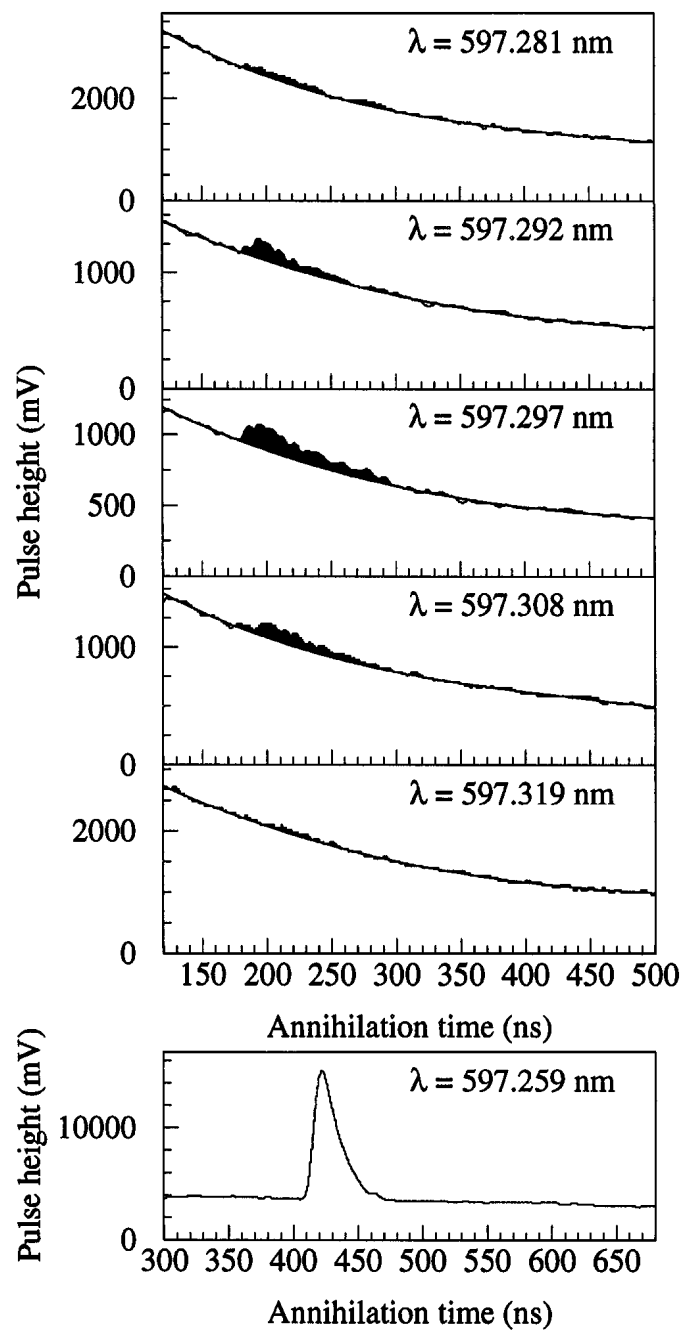

FIG. 2. Analog DATS (photomultiplier output pulse shape recorded in a digital oscilloscope) obtained with laser irradiation at different vacuum lengths $\lambda$ around $597.3 \mathrm{~nm}$ at $30 \mathrm{~K}$ and 5 bar with an $\mathrm{H}_{2}$ admixture of $100 \mathrm{ppm}$. The small peak appearing as the laser wavelength approaches $597.297 \mathrm{~nm}$ indicates laser-induced annihilations almost simultaneously with the laser firing time $(\sim 180 \mathrm{~ns})$. For comparison, the lowest panel shows the resonance spike of a "conventional" laser transition $[(n, l)=(39,35) \rightarrow(38,34)]$ from a metastable to a short-lived state in pure helium.

Assuming that the selective quenching of high- $n$ states observed for the $v=2$ and $v=3$ cascades applies to the $v=1$ and $v=0$ cases as well, we also tried to find the transitions $(n, l)=(38,36) \rightarrow(39,37)$ and $(38,37) \rightarrow(39,38)$ as laser resonances. The resonance profiles of these transitions, observed by slightly increasing the $\mathrm{H}_{2}$ concentration, are shown in Figs. 3(b) and 3(c), respectively.

Now that all the inverse transitions between $n=38$ and $n=39$ had been identified as laser resonances, the next step was to look for inverse transitions between $n=$ 37 and 38. Since it was known that lower- $n$ states are more resistant to collisional quenching by $\mathrm{H}_{2}$ molecules

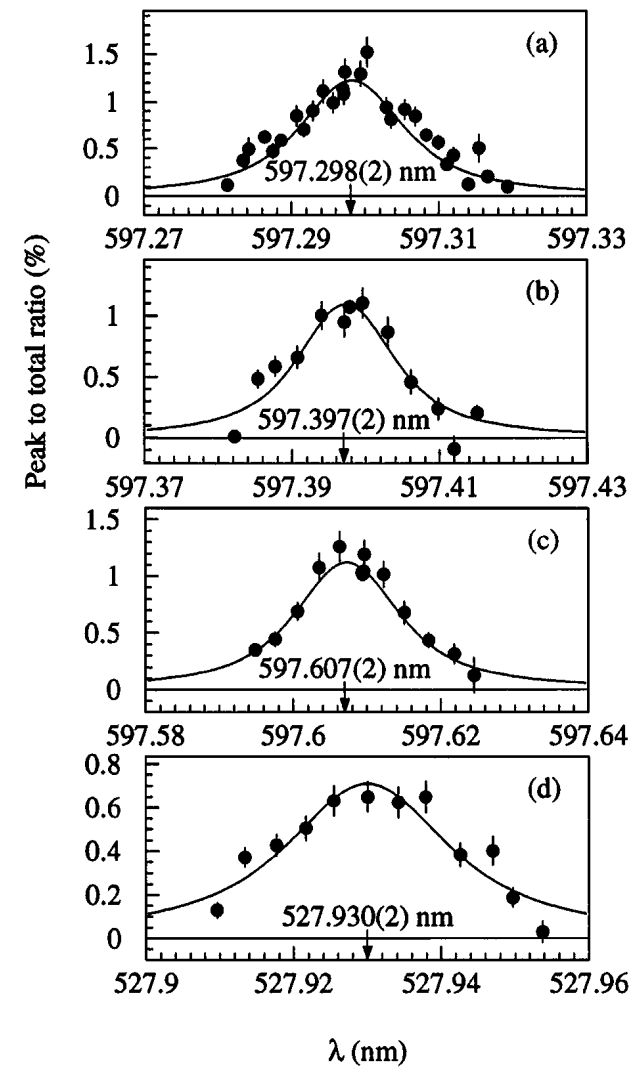

FIG. 3. Resonance profiles for those transitions, where wavelength scans were performed: (a) $(38,35) \rightarrow(39,36)$, (b) $(38,36) \rightarrow(39,37)$, (c) $(38,37) \rightarrow(39,38)$, and (d) $(37,36) \rightarrow$ $(38,37)$. The central wavelengths obtained by fitting a convolution of a Gaussian function with a Lorentzian to the data are also shown.

than higher- $n$ states, the $\mathrm{H}_{2}$ concentration was increased by a factor of $\sim 5-10$. The inverse resonance for the known 529.622(3) nm transition $(38,35) \rightarrow(37,34)$ [5] was immediately observed. Then, without scanning the laser wavelength, the transition $(37,35) \rightarrow(38,36)$ was also observed as a laser-induced peak similar to the one in Fig. 2 at the wavelength predicted by theory $[18,19]$. The resonance profile of the $v=0$ transition $(37,36) \rightarrow$ $(38,37)$ is shown in Fig. 3(d).

All six transitions observed by the HAIR method are summarized in the partial level scheme of $\bar{p}^{4} \mathrm{He}^{+}$displayed in Fig. 4. The experimental results for the wavelengths of these transitions are given in Table I. The errors of the experimental wavelengths arise mainly due to uncertainties in the wavelength calibration and the bandwidth of the laser pulse. For comparison, theoretical predictions for these wavelengths $[18,19]$ including relativistic corrections are also shown. These values are found to agree with the experimental results within a few ppm for the $v=0$ and 1 states as well as for the $v=2,3$, and 4 states [5,6].

To be able to detect laser resonances as visible peaks in the analog DATS, we had to find a suitable $\mathrm{H}_{2}$ concentration for each transition separately, which provided the 


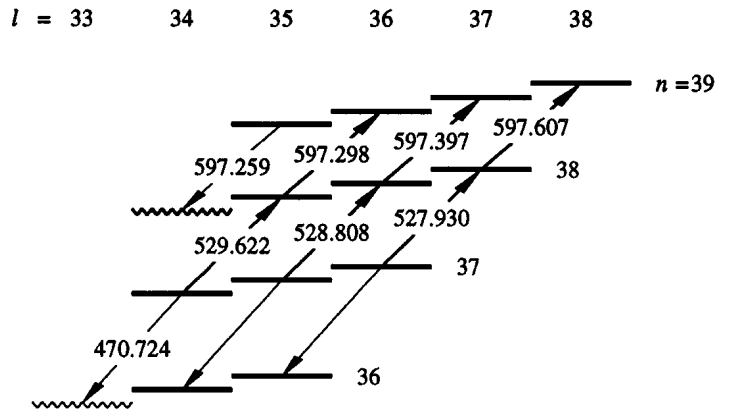

FIG. 4. Partial level scheme of $\bar{p}^{4} \mathrm{He}^{+}$, summarizing the six transitions between normally metastable states observed by the new HAIR method (bold arrows). Only the vacuum wavelengths for transitions observed until now are shown (in units of $\mathrm{nm}$ ).

balance between a short lifetime of the upper level and a sufficiently large population left in the lower level. In order to observe the $v=0$ transition from $n=38$ to 39 with the same intensity as the $v=2$ transition, about three times the amount of $\mathrm{H}_{2}$ had to be added. This fact reveals a weak $l$ dependence of the quenching strength by $\mathrm{H}_{2}$ molecules: states with the same principal quantum number $n$ but higher angular momentum number $l$ are less sensitive to quenching by $\mathrm{H}_{2}$ molecules. On the other hand, the strong $n$ dependence of the quenching observed for the $v=2$ and 3 cascades has been confirmed for the $v=1$ and $v=0$ cascades by the observation of $\mathrm{H}_{2}$ assisted inverse laser resonances in these cascades.

In summary, we have established a method to selectively shorten the lifetimes of specific metastable states of $\bar{p} \mathrm{He}^{+}$by adding an appropriate number of $\mathrm{H}_{2}$ molecules to the target gas. Since the laser resonance technique is applied to transitions from a metastable state to a shortlived one, the HAIR method allows all initially populated states to be accessed, not only the last one in each cascade. This was demonstrated by observing all the transitions $n=38 \rightarrow 39$ and $n=37 \rightarrow 38$ as laser resonances, including the first two transitions in the $v=0$ cascade between circular orbits. In addition to providing a way to find previously inaccessible transitions this method is also capable of giving important information on the $l$ and $n$ dependence of the $\mathrm{H}_{2}$ quenching of states. This will be treated in a separate publication [15]. Finally, now that we have established a way to access all metastable states of $\bar{p} \mathrm{He}^{+}$, the determination of the initial populations in these states seems to have come within the reach of this experiment.

We are indebted to the LEAR and PS staff at CERN for their tireless dedication to providing us with the highquality antiproton beam, to V.I. Korobov for making his
TABLE I. Experimental results $\lambda_{\exp }$ for the transition wavelengths of all six transitions observed by the HAIR method, compared with theoretical predictions $\lambda_{\text {theo }}$ by Korobov including relativistic corrections $[18,19]$. The transitions $(37,34) \rightarrow$ $(38,35)$ and $(37,35) \rightarrow(38,36)$ were observed as resonance spikes at the predicted wavelengths, but no wavelength scan was performed. The errors for these transitions correspond to half the expected widths (FWHM) of the resonance profiles.

\begin{tabular}{cccc}
\hline \hline$\left(n_{i}, l_{i}\right) \rightarrow\left(n_{f}, l_{f}\right)$ & $v$ & $\lambda_{\exp }[\mathrm{nm}]$ & $\lambda_{\text {theo }}[\mathrm{nm}]$ \\
\hline$(38,35) \rightarrow(39,36)$ & 2 & $597.298(2)$ & 597.298 \\
$(38,36) \rightarrow(39,37)$ & 1 & $597.397(2)$ & 597.398 \\
$(38,37) \rightarrow(39,38)$ & 0 & $597.607(2)$ & 597.609 \\
\hline$(37,34) \rightarrow(38,35)$ & 2 & $529.622(8)$ & 529.623 \\
$(37,35) \rightarrow(38,36)$ & 1 & $528.808(8)$ & 528.808 \\
$(37,36) \rightarrow(38,37)$ & 0 & $527.930(2)$ & 527.931 \\
\hline \hline
\end{tabular}

theoretical results available to us, and to Hamamatsu Photonics Ltd. for developing a special gated PMT used in this experiment. The present work is supported by the German Bundesministerium für Bildung, Wissenschaft, Forschung und Technologie, the Grants-in-Aid for Specially Promoted Research and for International Scientific Research of the Japanese Ministry of Education, Science and Culture, and the Hungarian National Science Foundation. H. A. T. acknowledges the support of the Japan Society for the Promotion of Science.

[1] N. Morita et al., Phys. Rev. Lett. 72, 1180 (1994).

[2] R. S. Hayano et al., Phys. Rev. Lett. 73, 1485 (1994); 73, 3181(E) (1994).

[3] F. E. Maas et al., Phys. Rev. A 52, 4266 (1995).

[4] H. A. Torii et al., Phys. Rev. A 53, R1931 (1996).

[5] R. S. Hayano et al., Phys. Rev. A 55, R1 (1997).

[6] T. Yamazaki et al. (to be published).

[7] M. Iwasaki et al., Phys. Rev. Lett. 67, 1246 (1991).

[8] T. Yamazaki et al., Nature (London) 361, 238 (1993).

[9] S. N. Nakamura et al., Phys. Rev. A 49, 4457 (1994).

[10] E. Widmann et al., Phys. Rev. A 51, 2870 (1995).

[11] B. Ketzer et al., Phys. Rev. A 53, 2108 (1996).

[12] E. Widmann et al., Phys. Rev. A 53, 3129 (1996).

[13] N. Morita, K. Ohtsuki, and T. Yamazaki, Nucl. Instrum. Methods Phys. Res., Sect. A 330, 439 (1993).

[14] T. Yamazaki et al., Chem. Phys. Lett. 265, 137 (1997).

[15] B. Ketzer et al. (to be published).

[16] M. Hori et al. (to be published).

[17] A. Niestroj et al., Nucl. Instrum. Methods Phys. Res., Sect. A 373, 411 (1996).

[18] V. I. Korobov, Phys. Rev. A 54, R1749 (1996).

[19] V.I. Korobov, in Proceedings of the International Conference on Low Energy Antiproton Physics, Dinkelsbühl, 1996 (to be published). 